\title{
A case of isolated rectal recurrence of muscle invasive bladder
}

\section{cancer}

\author{
Shaqil N. Kassam, MD, MSc; ; Zared Aziz, MD, FRCPC; '† Lick San Hung, MD, FRCPC; ${ }^{\dagger}$ \\ Srikala S. Sridhar, MD, MSc, FRCPC
}

*Department of Medical Oncology and Hematology, Princess Margaret Hospital, Toronto, ON; †Department of Laboratory Medicine and Pathobiology, University of Toronto, Toronto, ON

Cite as: Can Urol Assoc J 2013;7(5-6):e376-80. http://dx.doi.org/10.5489/cuaj.1223 Published online May 13, 2013.

\section{Abstract}

We present the case of a 53-year-old man with a 25 -pack/year smoking history and a 6-month history of gross hematuria, who presented with a pT3a, N0, M0, muscle invasive bladder cancer (MIBC). He declined neoadjuvant chemotherapy, but received post-cystectomy adjuvant chemotherapy. Six months post-adjuvant chemotherapy, he presented with abdominal pain and a large bowel obstruction, and was found to have an isolated rectal recurrence of MIBC. This case illustrates 2 important issues: (1) patients with a smoking history and symptoms of hematuria need to be carefully evaluated to rule out urothelial cancer; and (2) in patients with muscle invasive disease, local pelvic recurrence is common and close surveillance for recurrence needs to be implemented.

\section{Case report}

A 53-year-old previously healthy male presented to his family physician with painless, intermittent, gross hematuria for the preceding 6 months and was referred for urological workup. Cystoscopy on initial presentation was suspicious for intramural thickening and induration of the right lateral bladder wall, with questionable involvement of the right ureteric orifice. A transurethral resection of a bladder tumour (TURBT) was performed and confirmed poorly differentiated transitional cell (TCC) with extensive invasion of the fibrous lamina propria and muscularis mucosa, but questionable involvement of the muscularis propria. Due to the uncertainty of muscle invasion, a repeat TURBT was performed which confirmed high-grade TCC with extensive invasion into the muscularis propria. Subsequent staging investigations, including computed tomography (CT) and bone scan, showed thickening of the right lateral bladder wall with possible involvement of the distal right ureter, but no evidence of distant metastatic disease. Due to the invasive nature of his bladder cancer and the known survival benefit with neoadjuvant chemotherapy, ${ }^{1}$ the patient was seen at our tertiary cancer centre for consideration of neoadjuvant chemotherapy. The patient declined neoadjuvant chemotherapy and chose to proceed with cystectomy with adjuvant chemotherapy if required.

He underwent radical cystoprostatectomy and distal ureteric resection, pelvic lymph node dissection (distal to the external iliacs), and creation of an ileoconduit. Frozen sections at the time of surgery showed no involvement of the distal ureters. Final pathology revealed high-grade TCC with invasion of the muscularis propria and perivesical tissue both anteriorly and posteriorly (Fig. 1). The tumour also showed extensive lymphatic and perineural invasion with tumour in the lymphatics around the left seminal vesicle, but not involving the seminal vesicles, prostate or urethra; and 5 of the 17 lymph nodes were positive for TCC. The case was presented at our Multidisciplinary Cancer Conference (MCC) where it was agreed that the best course of action would be to proceed with 6 cycles of adjuvant gemcitabine and cisplatin chemotherapy and then consider pelvic radiation due to the extensive involvement of the disease. The patient completed 6 cycles of chemotherapy, but did not receive any radiation therapy following chemotherapy.

Unfortunately, 6 months after completing chemotherapy the patient presented to the emergency department with anorexia, diffuse abdominal pain and pencil thin stools. The patient was booked for urgent magnetic resonance imaging (MRI) of the abdomen and pelvis. This showed circumferential thickening of the rectum (Fig. 2), suspicious for metastatic TCC, but a metachronous primary rectal cancer could not be ruled out. Subsequent colonoscopy revealed a fixed pelvis with rectosigmoid mucosal induration and thickening. Rectal biopsies confirmed poorly differentiated carcinoma favouring high-grade TCC (Fig. 3). Given the rarity of isolated rectal recurrence, the patient was again presented at the MCC where it was suggested that given his poor performance status, symptom control with a rectal stent would be the best course of action, and that further invasive treatment would only impart risk and morbidity with 


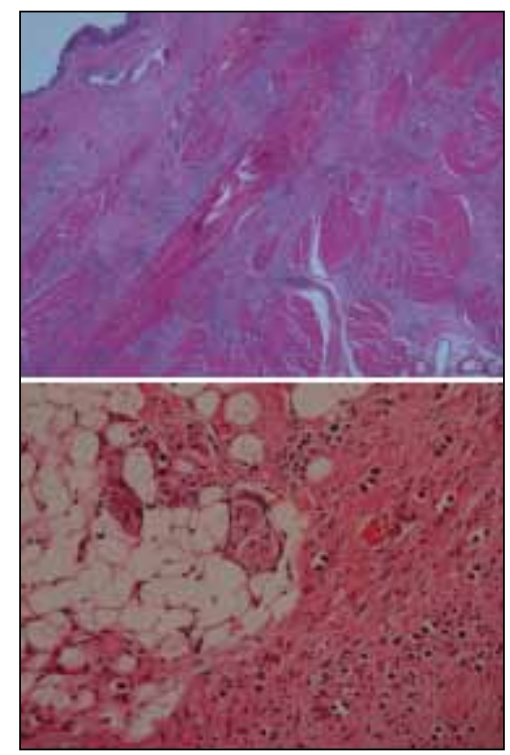

Fig. 1. Pathology sections showing small, hyperchromatic and mildly pleomorphic tumour cells (i) invading the bladder wall (2x) and (ii) present within the perivesical adipose tissue (20x). minimal benefit to the patient. With the patient's approval, outpatient palliative care was organized to maximize the patient's quality of life. Unfortunately, about 23 months after being diagnosed with pT3a bladder cancer, and 6 months after being diagnosed with rectal recurrence, the patient died from progressive disease and urosepsis.

\section{Discussion}

Gross hematuria, in addition to being alarming to the patient, can herald malignancy in up to $20 \%$ to $40 \%$ of cases, ${ }^{2}$ and therefore requires prompt investigation under the appropriate clinical circumstances. The source of hematuria can originate from any point in the urinary system, extending from the kidney and upper tracts to the urethral meatus; therefore, this situation requires evaluation of the entire tract to determine the exact cause of bleeding. Fortunately, the differential diagnosis for hematuria is relatively narrow, with the most common causes in adults being urinary tract infections, neoplasms and urolithiasis. ${ }^{3}$ However, since the misdiagnosis of gross hematuria can have devastating consequences, it is important to identify and appropriately investigate those patients at high risk for a neoplastic cause of their hematuria (Table 1). ${ }^{4}$ Any individual with gross hematuria and risk factors for urological malignancy should be referred to a urologist for complete urinary tract evaluation. ${ }^{3}$ Current guidelines suggest that urinalysis with microscopy and urine cytology be the initial workup, followed by cystoscopy and triphasic CT urogram under the guidance of a urologist. ${ }^{3}$

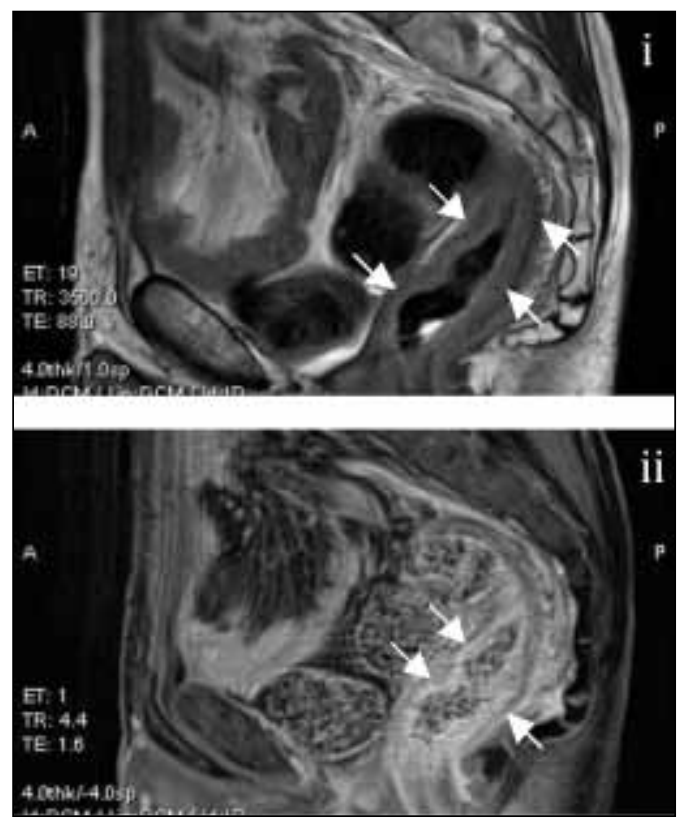

Fig. 2. Sagittal magnetic resonance images; (i) pre- and (ii) post-administration of gadolinum showing mild diffuse rectal wall thickening.

As illustrated by this case, the traditional mainstay of treatment of MIBC is radical cystectomy with pelvic lymphadenectomy. However, there has been a recent treatment paradigm shift as there is now mounting long-term evidence that suggests cisplatin based neoadjuvant chemotherapy confers an overall survival advantage of between $5 \%$ to $8 \%{ }^{5,6}$ This change has been adopted in several guideline statements as grade A recommendations, where the inclusion of cisplatin based neoadjuvant chemotherapy for MIBC should, at the very least, be offered to patients. ${ }^{7}$ Unfortunately our patient, along with many others in his situation, are offered and decline neoadjuvant chemotherapy or are not even offered neoadjuvant chemotherapy possibly due to the stigma and concerns about toxicity associated with chemotherapy, or due to the potential delay in receiving definitive surgical treatment. In either case, better education about the controllable side effects of chemotherapy and the potential survival benefit of neoadjuvant chemotherapy needs to be conveyed to patients in an attempt to decrease the mortality and the recurrence rate of MIBC.

Recurrence of MIBC can be distant recurrence (DR) and local recurrence (LR). Although MIBC can metastasize to any location, the most common sites of distant, non-lymph node recurrence is bone, liver and lung. ${ }^{8}$ Local recurrence of MIBC on the other hand, as defined in the literature, encompasses tumour recurrence in the pelvic soft-tissue and/ or lymph nodes distal to the external iliac bifurcation, and in some studies, distal to the aortic bifurcation..$^{18}$ LR of muscle invasive bladder cancer after radical cystectomy and pelvic 


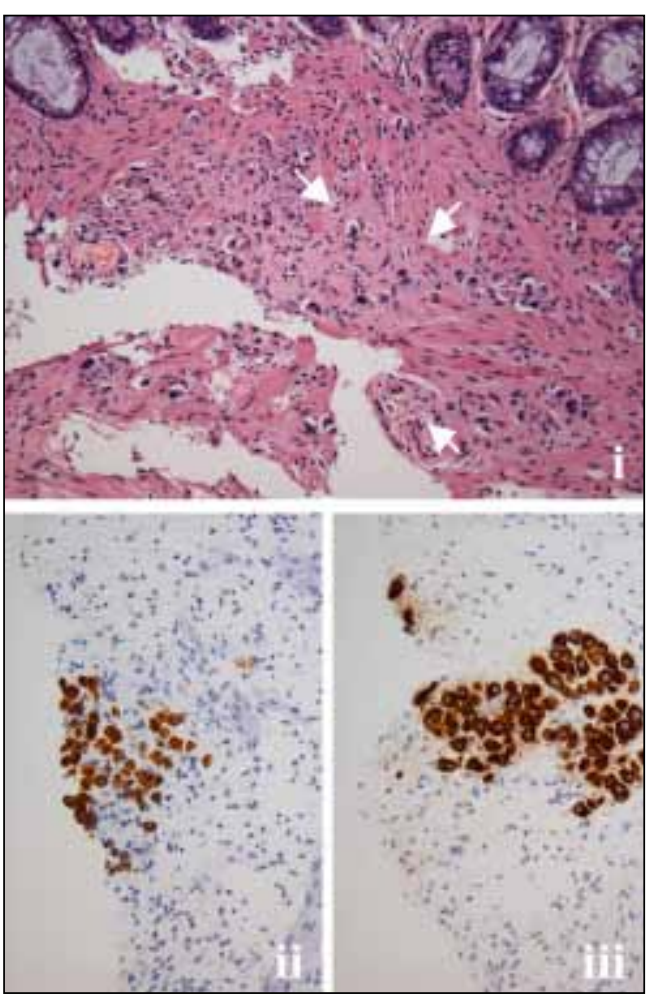

Fig. 3. Rectal biopsy sections showing (i) poorly differentiated tumour cells within the submucosa and sparing the mucosa; (ii) combined with CK7 positivity (iii) and CK 20 strongly suggests colorectal metastasis of urothelial origin, as opposed to primary colon cancer.

lymph node dissection was thought to be rare prior to the routine use of CT in cancer surveillance. ${ }^{8,10}$ Since then, the rates of $L R$ involving the pelvis after cystectomy for bladder cancer are between $16 \%$ and $19 \%$ (Table 2, column A), with $63 \%$ of these associated with pathologically extravesical disease. ${ }^{10}$ Contemporary series, however, define LR as recurrence isolated to the pelvis or to exclude patients with pelvic recurrence with concurrent metastatic involvement outside of the pelvis. ${ }^{11}$ In these cases, the isolated pelvic LR rate is reported between $5 \%$ and $7 \%$ (Table 1, column B). Although imaging has greatly enhanced the diagnosis of $L R$, most recurrences are only detected when the patient becomes symptomatic, ${ }^{5,11}$ which may suggest that current surveillance strategies do a poor job of detecting LR because LR after cystectomy tends to occurs relatively early (Table 1, column C).

Although the rate of pelvic recurrence after radical cystectomy is relatively common (Table 1 , column B), there is a paucity of information regarding the rate of isolated rectal recurrence post-cystectomy. In the study by Honma and colleagues, $55 \%$ of patients with isolated pelvic recurrence had recurrence within the pelvic soft tissue in front of the anterior rectal wall. The study did not comment on recurrence involving the rectum specifically. ${ }^{10}$ In another
Table 1. Risk factors for a neoplastic cause of hematuria A smoking history

An occupational exposures to chemicals including benzenes and aromatic amines

Irritative voiding symptoms

Previous gross hematuria, previous genitourinary cancer

Previous pelvic irradiation

Patients who are 40 years of age or older

study, autopsies performed on (pT2-pT4) patients with MIBC treated with cystectomy showed that $12 \%$ to $13 \%$ of patients had some form of intestinal involvement, but again data on rectal recurrence was not reported..$^{15,16}$ Most of the literature regarding rectal recurrence of muscle invasive bladder cancer comes from isolated case reports ${ }^{15,17}$ and autopsy-based studies, which describe rectal recurrence affecting about $10 \%$ of patients with LR post-cystectomy for muscle-invasive bladder cancer. ${ }^{18}$ Indeed this has been confirmed by a recent retrospective study using transrectal ultrasonagraphy to detect local recurrence after cystecomy, which found the rates of rectal recurrence in patients with pelvic local recurrence of bladder cancer to be as high as $9.7 \% .^{11}$

Despite these high rates of recurrence of $\mathrm{MIBC}$, there is an absence of robust evidence to guide surveillance of these patients after surgical intervention. A recent retrospective study based on the recurrence patterns of 2287 patients across Canada advocates for a pathological stage based surveillance protocol, with higher stage disease being investigated earlier and more aggressively. ${ }^{12}$ The subject of surveillance also identifies a unique issue illustrated by this case, namely that of cisplatin resistance. Data from the cisplatin sensitive Germ Cell Tumour (GCT) literature defines cisplatin resistance by the time to cancer relapse after completion of cisplatin based chemotherapy. Early relapse is defined by relapse within 2 years after completion of therapy, while cisplatin refractory disease is defined as relapse while on cisplatin treatment or within 4 weeks after its completion. ${ }^{13}$ The treatment options for cisplatin resistant MIBC are currently limited, and overall outcomes are poor. ${ }^{14} \mathrm{~A}$ case could be made for an aggressive surgical approach similar to that used in cisplatin resistant $\mathrm{GCTs},{ }^{13}$ unfortunately in this circumstance our patient was not a surgical candidate due to his poor functional status. Another question arising from this case is the role of adjuvant radiation in patients at high risk for local failure post-cystectomy. Patients postneoadjuvant chemotherapy and surgery with residual disease could undergo additional radiation therapy to improve pelvic control and reduce morbidity associated with pelvic recurrences. Although not a standard approach, adjuvant radiotherapy in MIBC warrants further investigation. 
Table 2. Rates of and median time to local recurrence after treatment of muscle invasive bladder cancer treated with cystectomy and lymphadenectomy without adjuvant treatment

A

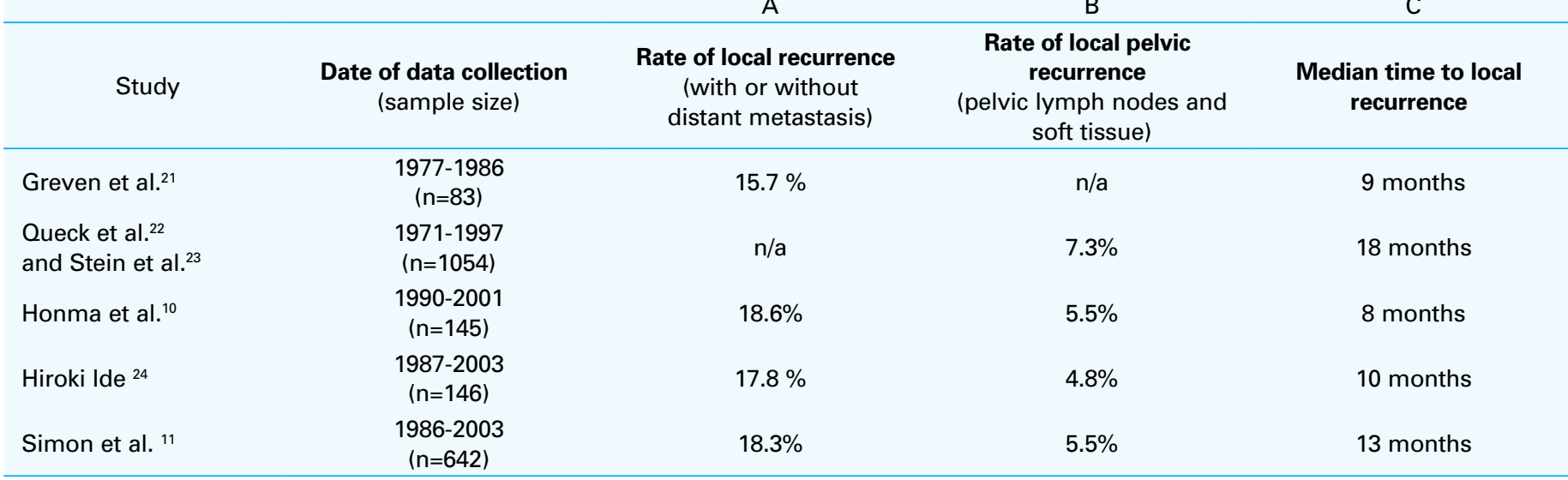

\section{Conclusion}

We illustrated some of the diagnostic and treatment related issues related to MIBC, specifically the need to investigate hematuria in the right clinical context and to offer neoadjuvant cisplatin based chemotherapy to all patients with MIBC. We also demonstrated that isolated pelvic local recurrence after muscle invasive bladder cancer is relatively common, and that the rates of rectal recurrence may also be higher than once thought. Taken together, the literature suggests that about $0.5 \%$ of all patients with MIBC will have rectal recurrence, and that this recurrence will likely be diagnosed after the patient becomes symptomatic with symptoms of bowel obstruction. ${ }^{9,15,17}$ This signifies a large burden of morbidity in North America as 7100 patients in Canada and 70530 in the United States were diagnosed with bladder cancer in 2010 alone. ${ }^{19,20}$ Given that the mean survival after diagnosis of rectal recurrence of MIBC is about 5 months, an evidence-based surveillance regimen is also required to mitigate the significant mortality associated with MIBC recurrence.

Competing interests: None declared.

This paper has been peer-reviewed.

\section{References}

1. Advanced Bladder Cancer Meta-analysis Collaboration. Neoadjuvant chemotherapy in invasive bladder cancer: a systematic review and meta-analysis. Lancet 2003;36:1927-34.

2. Ismail M, Gomella LG. Hematuria: Evaluation and management. In: Urology for Primary Care Physicians. Philadelphia, PA: W.B. Sunders; 1999:59-74.
3. Patel JV, Chambers CV, Gomella LG. Hematuria: etiology and evaluation for the primary care physician. Can J Urol 2008;62 (Suppl 1):54-61.

4. Grossfeld GD, Wolf IS Jr, Litwan MS, et al. Asymptomatic microscopic hematuria in adults: summary of the AUA best practice policy recommendations. Am Fam Phys 2001;63:1145-54.

5. Sherif A, Holmberg L, Rintala E, et al. Neoadjuvnt cisplatin based combination chemotherapy in patients with invasive bladder cancer: a combined anylysis of two Nordic Studies. Eur Urol 2004;45:297-303. http://dx.doi.org/10.1016/i.eururo.2003.09.019

6. Advanced Bladder Cancer Meta-analysis Collaboration. Adjuvant chemotherapy in invasive bladder cancer: a systematic review and meta-analysis of individual patient data. Eur Urol 2005;48:189-201. http:// dx.doi.org/10.1016/i.eururo.2005.04.005

7. Stenzl A, Cowan NC, De Santis M, et al. Treatment of Muscle-invasive and Metastatic Bladder Cancer: Update of the EAU Gudelines. Eur Urol 2011;59:1009-18. http://dx.doi.org/10.1016/i.eururo.2011.03.023

8. Shinagare AB, Ramaiya NH, Ragannathan JP, et al. Metastatic pattern of bladder cancer: correlation with the characteristics of the primary Tumor. AJR 2011;196:117-22. http://dx.doi.org/10.2214/ AJR. 10.5036

9. Cagiannos I, Morash C. Surveillance strategies after definitive therapy of invasive bladder cancer. Can Urol Assoc J 2009;3:S237-42.

10. Honma I, Masumor N, Sato E, et al. Local recurrence after radical cystectomy for invasive blader cancer: an analysis of predictive factors. Urology 2004;64:744-8. http://dx.doi.org/10.1016/i.urology.2004.05.003

11. Simon J, Bartsch G, Rinnab L, et al. Transrectal Ultrasound as Diagnostic tool for the detection of local recurrence following cystectomy and urinary diversion. Urol Int 2009;82:12-6. http://dx.doi. org/10.1159/000176018

12. Slaton JW, Swanson DA, Grossman HB, et al. A stage specific approach to tumor surveillance after radical cystectomy for transitional cell carcinoma of the bladder. J Urol 1999;162:710-4. http://dx.doi. org/10.1097/00005392-199909010-00021

13. Koychev D, Oechsle K, Bokemeyer C, et al. Treatment of patients with relapsed and/or cispltin refractory metastatic germ cell tumours: an update. Int J Androl 2011;34:266 -73. http://dx.doi.org/10.1111/ j. $1365-2605.2011 .01145 . x$

14. Tsai $\mathrm{YC}, \mathrm{Hsu} \mathrm{CH}$, Huang $\mathrm{CY}$, et al. Gemcitabine and ifosfamide as a second-line treatment for cisplatinrefractory metastatic urothelial carcinoma: a phase II study. Anti-cancer Drugs 2007;18:487-91. http:// dx.doi.org/10.1097/CAD.0b013e3280126603

15. Langenstroer $P$, Zacharias $A$, Almagro $U$, et al. Annular constriction of the rectum secondary to transitional cell carcinoma of the bladder. Urology 1996;47:442-4. http://dx.doi.org/10.1016/500904295(99)80471-3

16. Wallmeroth A, Wagner U, Moch H, et al. Patters of metastasis in muscle-invasive bladder cancer (pT2-4):An autopsy study on 367 patients. Urol Int 1999;62:69-75. http://dx.doi.org/10.1159/000030361

17. Stillwell TJ, Rife CC, Lieber MM. Bladder carcinoma presenting with rectal obstruction. Urology 1989;34:238-40. http://dx.doi.org/10.1016/0090-4295(89)90315-4

18. Saitoh $H$, Hida M, Wakabayashi $T$, et al. Metastasis of urothelial tumors of the bladder: correlation between sites and number of organs involved. Tokai J Exp Clin Med 1982;7:517-26. 
Kassam et al.

19. Canadian Cancer Society's Steering Committee: Canadian Cancer Statistics 2010. Toronto: Canadian Cancer Society 2010. http://www.cancer.ca. Accessed May 2, 2013.

20. American Cancer Society. Cancer facts \& figures 2010. Atlanta: American Cancer Socieity 2010. Available at http://www.cancer.org/acs/groups/content/@epidemiologysurveilance/documents/document/ acspc-026238.pdf. Accessed May 2, 2013.

21. Greven KM, Spera JA, Solin $\mathrm{U}$ et al. Local recurrence after cystectomy alone for bladder carcinoma Cancer 1992;69:2767-70. http://dx.doi.org/10.1002/1097-0142(19920601)69:11<2767::AlDCNCR2820691123>3.0.C0;2-\#

22. Quek ML, Stein JP, Clark PE, et al. Natural history of surgically treated bladder carcinoma with extravesical tumor extension. Cancer 2003;98:955-61. http://dx.doi.org/10.1002/cncr.11569
23. Stein JP, Lieskovsky $\mathrm{G}$, Cote $\mathrm{R}$, et al. Radical cystectomy in the treatement of invasive bladder cancer; long term results in 1,054 patients. J Clin Oncol 2001;19:666-75.

24. Ide H, Kikuchi E, Miyaijma A, et al. The predictors of local recurrence after radical cystecomy in patients with invasive bladder cancer. Jpn J Clin Concol 2008;38:360-4. http://dx.doi.org/10.1093/iico/hyn036

Correspondence: Dr. Srikala S. Sridhar, University Health Network, Princess Margaret Hospital, 5-222 610 University Ave., Toronto, 0N M5G 2M9; srikala.sridhar@uhn.ca 